\begin{tabular}{|c|l|}
\hline Title & Matrix sublimation method for the formation of high-density amorphous ice \\
\hline Author(s) & Kouchi, A.; Hama, T.; Kimura, Y.; Hidaka, H.; Escribano, R.; W atanabe, N. \\
\hline Citation & $\begin{array}{l}\text { Chemical physics letters, 658, 287-292 } \\
\text { https://doi.org/10.1016/.cplett.2016.06.066 }\end{array}$ \\
\hline Issue Date & 2016-06-24 \\
\hline Doc URL & http://hdl.handle.net/2115/70840 \\
\hline Rights & $\begin{array}{l}\text { O2016, Elsevier. This manuscript version is made available under the CC-BY-NC-ND 4.0 license } \\
\text { http://creativecommons.org/icenses/by-nc-nd/4.0/ }\end{array}$ \\
\hline Rights(URL) & http://creativecommons.org/icenses/by-nc-nd/4.0/ \\
\hline Type & Kouchi et al. CPL 03.text.pdf \\
\hline article (author version)
\end{tabular}

Instructions for use 


\section{Matrix sublimation method for the formation of high-density amorphous ice}

2

3 Kouchi, A. ${ }^{\mathrm{a}}{ }^{*}$, Hama, T. ${ }^{\mathrm{a}}$, Kimura, Y. ${ }^{\mathrm{a}}$, Hidaka, H. ${ }^{\mathrm{a}}$, Escribano, R. ${ }^{\mathrm{b}}$, \& Watanabe, N. ${ }^{\mathrm{a}}$

4

$5 \quad{ }^{a}$ Institute of Low Temperature Science, Hokkaido University, Sapporo 060-0819, Japan.

6 b Instituto de Estructura de la Materia, Consejo Superior de Investigaciones Cientificas,

7 IEM-CSIC, 28006 Madrid, Spain.

$8 *$ Corresponding author.

$9 \quad$ E-mail address: kouchi@lowtem.hokudai.ac.jp (A. Kouchi)

10

11 Graphical abstract

12

13 Highlights

14 - Matrix sublimation method for preparing amorphous ice has been developed.

15 - Amorphous ice formed exhibits a highly porous microscale texture.

16 - The amorphous ice is a high-density form similar to that formed at high-pressures. 
18 Keywords: Matrix sublimation, ice, high-density amorphous ice,

20 ABSTRACT

21 A novel method for the formation of amorphous ice involving matrix sublimation has

22 been developed. A CO-rich $\mathrm{CO}: \mathrm{H}_{2} \mathrm{O}$ mixed ice was deposited at 8-10 K under ultra-high

23 vacuum condition, which was then allowed to warm. After the sublimation of matrix

$24 \mathrm{CO}$ at $35 \mathrm{~K}$, amorphous ice remained. The amorphous ice formed exhibits a highly

25 porous microscale texture; however, it also rather exhibits a density similar to that of

26 high-density amorphous ice formed under high pressure. Furthermore, unlike

27 conventional vapor-deposited amorphous ice, the amorphous ice is stable up to $140 \mathrm{~K}$,

28 where it transforms directly to cubic ice Ic.

1. Introduction

The importance of amorphous ice is widely recognized in the fields of physics, 
33 chemistry [1,2], and cryobiology [3], but is of particular interest in planetary science

34 and astrophysics [4]. Various procedures for the preparation of amorphous ice have been

35 developed in order to investigate the relationship between its structure and method of

36 formation [2] (Fig. 1). Vapor deposition methods were often applied to prepare

37 amorphous solid water (ASW) in a vacuum [5,6]. When water $\left(\mathrm{H}_{2} \mathrm{O}\right)$ vapor is deposited

38 onto a surface below $40 \mathrm{~K}$, high-density ASW can be produced. In contrast, deposition

39 at $40-70 \mathrm{~K}$ results in low-density ASW [6,7]. Unique property of ASW that is distinct

40 from other amorphous ices is that ASW has larger surface area and higher porosity $[8,9]$.

41 Rapid cooling of micron-sized water droplets or thin film can form amorphous ice

42 referred to as hyperquenched glassy water (HGW) [10,11]. HGWs formed at ambient

43 and high pressures are low- and high-density forms, respectively [12].

44 Disruption of ice crystals by compression or irradiation with high-energy particles is also known as formation method of amorphous ice [13-16]. High-density amorphous ice

46 (HDA), which is distinct from the ASW and HGW formed at low pressures, could be

47 formed by compression of ice crystals at high pressure [13,14]. HDA transforms 
49 vacuum can be amorphized by the irradiation with high-energy electron beam [15] or

$50 \quad$ UV-rays at low temperature [16].

51 Since the structure of amorphous ice has been found to strongly depend on the

52 method of its formation [2,17-19] and formation conditions [2,6,7,12], as described

53 above, unknown amorphous ice structures may still be discovered through new

54 formation methods. In this letter, we report a new method for the formation of

55 amorphous ice, matrix sublimation method. Remarkably, ice prepared by sublimation of

56 a carbon monoxide (CO) matrix from a $\mathrm{H}_{2} \mathrm{O} / \mathrm{CO}$ mixture at around $35 \mathrm{~K}$ shows a highly

57 porous texture different from that of ASW, and a density similar to that of HDA

58 prepared under high pressure. This new method contributes to our understanding of the

59 structure and physical properties of amorphous ice and to the development of new

60 materials.

61

62

\section{Experimental}

63

64 Amorphous ice was prepared according to a newly developed matrix sublimation 
method, illustrated in Fig. 2. We observed this process in situ using the ultra-high

vacuum (UHV) transmission electron microscope (TEM) and Fourier-transform

67 infrared (FTIR) spectrometry. was developed following Kondo et al. [20]. Since the specimen was surrounded by a

70 liquid nitrogen shroud, the pressure around the specimen was lower than the pressure of

71 the specimen chamber $\left(1 \times 10^{-6} \mathrm{~Pa}\right)$. We used a liquid He cooling holder (Gatan 72 ULTST) for specimen cooling, and a 5-nm-thick amorphous Si film (SiMPore Inc.) as a

73 substrate for sample deposition. Of the three ports directed at the specimen surface, the

74 one with an incident angle of $55^{\circ}$ was used for a Ti gas inlet tube (inner diameter $=0.4$

$75 \mathrm{~mm})$.

76 CO was used as the matrix because it is an astrophysically important molecule,

77 active for IR spectroscopy, and could be evacuated by ion pumps equipped with a TEM.

78 Mixtures of $\mathrm{CO}$ and $\mathrm{H}_{2} \mathrm{O}$ at ratios of 50:1 and 10:1 were deposited on the Si thin film at

79 about $8 \mathrm{~K}$. We did not record TEM images during deposition to avoid damage from the

80 electron beam. After deposition, the sample substrate was warmed at a rate of 
81 approximately $2.0 \mathrm{~K} / \mathrm{min}$, controlled manually. TEM images could not be recorded

82 during the sublimation of $\mathrm{CO}$ because the pressure was higher than $10^{-4} \mathrm{~Pa}$. The

83 thickness of the ice samples remaining after CO sublimation was several tens of

84 nanometres. To avoid electron-beam damage to the sample, a low-dose imaging

85 technique was applied in which the accelerating voltage was $80 \mathrm{kV}$, the electron beam

86 current was less than $0.1 \mathrm{pA} / \mathrm{cm}^{2}$ and low magnification images were recorded using a

87 CCD camera (Gatan ES500W). Furthermore, subsequent observations were taken from

88 different positions of the sample. The edge of a single crystalline Si grid was used for

89 the calibration of length in the analysis of the electron diffraction patterns.

90 For FTIR spectroscopy, we used a laboratory setup for surface reactions in

91 interstellar environments (LASSIE) apparatus, as described previously [21]. The

92 samples were prepared using a background deposition method on an $\mathrm{Al}$ substrate cooled

93 to $10 \mathrm{~K}$ by He refrigeration. After deposition, the sample substrate was warmed at a rate

94 of $2.0 \mathrm{~K} / \mathrm{min}$. IR spectra of samples were obtained and monitored by

95 reflection-absorption spectroscopy. Typical column density of the samples after CO

96 sublimation was $1.5 \times 10^{16}$ molecules $/ \mathrm{cm}^{2}$. 


\section{Results and discussion}

$101 \square$ CO and a small amount of amorphous CO. Water molecules are embedded in the CO matrix as clusters (see Supplementary Fig. 1 and Supplementary Table 1. [22-24]). After

103 the sublimation of $\mathrm{CO}$ at 25-40 K, we observed highly porous ice, as shown in Fig. 3b

104 and Fig. 4; we term this the matrix-sublimated ice (MSI). The MSI shows wide textural

105 variety at the micro- and submicroscale, with the ice exhibiting mainly concrete-like

106 (Fig. 4a) or granite-like (Fig. 4c) textures, along with some minor occurrences of

107 feather-like (Fig. 4b) or seaweed-like (Fig. 4d) textures. Conversely, these types of

108 structures (micro- and submicroscale highly porous structures) are not observed in pure

$109 \mathrm{H}_{2} \mathrm{O}$ vapor-deposited amorphous ice, that is, ASW, as shown in Fig. 5. In addition, no

110 temperature dependence on the structure of ASW was observed until crystallization

111 occurs. However, the previous studies reported that ASW has larger surface area and

112 higher porosity at lower temperatures, and it becomes less porous as the temperature 
113 increases $[8,9]$. We speculate that the space scales of porous structures are different

114 between ASW (nanometer scale) and MS-HDA (micrometer scale), and the

115 magnification in the present observation by TEM is too low to explicitly observe the

116 porous structures of ASW. These results confirm that the formation of the highly porous

117 MSI observed in the present study is not due to electron beam irradiation during TEM

118 but due to intrinsic phenomena. This is further confirmed by IR measurements, in which

119 a destructive beam is not applied, as discussed later.

120 As described below, we have confirmed that the structure of our MSI is that of an

121 HDA ice, and have therefore termed it matrix-sublimated high-density amorphous

122 (MS-HDA) ice. Figure 6 shows the temperature dependence of the d-spacing in the

123 main halo for vapor-deposited ASW and MS-HDA ice. In ASW, the d-spaces of the

124 main peak increase with temperature, as observed by Jenniskens and Blake [6]. They

125 assigned the lower and higher temperature forms as high-density ASW (HD-ASW) and

126 low-density ASW (LD-ASW), respectively. Conversely, the d-spacing in the MS-HDA

127 ice does not change with temperature, and remains constant at ca. $0.31 \mathrm{~nm}$ irrespective

128 of whether the initial ice composition $\left(\mathrm{CO}: \mathrm{H}_{2} \mathrm{O}\right)$ is $10: 1$ or $50: 1$. Winkel et al. reported 
129 that the d-spaces in HDA ice made by submitting ice Ih to high pressure ranged from

1300.28 to $0.32 \mathrm{~nm}$ [18], and the d-spaces of $0.31 \mathrm{~nm}$ for MS-HDA ice fall within this

131 range. The density of MS-HDA ice is estimated to be $1.16 \mathrm{~g} / \mathrm{cm}^{3}$ from the relationships

132 of d-spaces with pressure (0.1 GPa) [18], and pressure with density [25,26]. Mishima et

133 al. have reported that the density of HDA ice at ambient pressure is $1.17 \mathrm{~g} / \mathrm{cm}^{3}$ [13].

134 Thus, we conclude that the MS-HDA ice generated in the present study is a high-density

135 form similar to HDA ice produced under high pressure, but different from HD-ASW

136 produced by vapor-deposition in a vacuum.

137 MS-HDA ice formed at around $35 \mathrm{~K}$ is very stable against temperature change up to

138140 K. At temperatures around 140 K, MS-HDA ice directly transforms into ice Ic (Fig.

139 7). The transformation of HDA ice during heating depends on the initial formation

140 conditions, annealing conditions, pressure and heating rate. Consequently, different

141 transformation sequences (e.g. HDA $\rightarrow$ LDA $\rightarrow$ ice Ic and HDA $\rightarrow$ ice Ic) have been

142 observed [27-29]. Although direct comparison between MS-HDA ice and HDA ice is

143 difficult, it is worth noting that the behaviour of MS-HDA ice observed in the present

144 study resembles that of HDA ice formed at 0.1 GPa [27] rather than that formed at 
146 Figure 8 and Supplementary Fig. 1 show the changes in the FTIR spectra with

147 temperature for ice of three different initial CO: $\mathrm{H}_{2} \mathrm{O}$ ratios, i.e. 50:1, 10:1 and pure $\mathrm{H}_{2} \mathrm{O}$

148 (ASW). A summary of the assignments is given in Supplementary Table 1. At low

149 temperatures (10 and $20 \mathrm{~K}$ ), monomers are present in the 50:1 ice, as the $v_{3}$ asymmetric

150 and $v_{1}$ symmetric stretching modes of $\mathrm{H}_{2} \mathrm{O}$ monomers are observed at $3707 \mathrm{~cm}^{-1}$ and

$1513617 \mathrm{~cm}^{-1}$, respectively. However, no monomers are indicated in the 10:1 ice, but strong

152 absorptions due to polymeric aggregates are observed (Supplementary Table 1). During

153 the sublimation of $\mathrm{CO}$ around $30 \mathrm{~K}$, the intensity of the water cluster bands decreases

154 suddenly, and the characteristic $\mathrm{OH}$ stretching band at $3000-3600 \mathrm{~cm}^{-1}$ of solid water

155 ice becomes stronger. After the sublimation of CO, MS-HDA ice remains. The spectral

156 characteristics of the $\mathrm{OH}$ stretching band clearly differ between MS-HDA ice and ASW

157 (Fig. 8). The spectral features and peak wavenumbers of MS-HDA ice made from the

$158 \mathrm{CO}: \mathrm{H}_{2} \mathrm{O}$ mixture at ratios of 50:1 (Figs. 8a and b) and 10:1 (Figs. 8c and d) do not

159 change with temperature, indicating the stability of MS-HDA ice. Conversely, those of vapor-deposited ASW changes as the temperature increases (Figs. 8e and f) [30], 
161 indicating that HD-ASW deposited at $10 \mathrm{~K}$ is relatively unstable. Dangling-OH bonds

162 were observed in the IR spectra of MS-HDA ice and vapor-deposited ASW

163 (Supplementary Fig. 2) [31]. These spectroscopic results imply that MS-HDA has

164 porous structures like ASW at nanometer scale, in addition to the porous structures at

165 micrometer scale as shown in Fig. 5.

166 Although we cannot precisely clarify the formation mechanism of MS-HDA ice, the

167 following rationales are presented: The Laplace pressure of a $1.0 \mathrm{~nm}$ radius fine particle

168 of MS-HDA ice is $0.14 \mathrm{GPa}$ if we assume that its surface tension $70 \mathrm{mN} / \mathrm{m}$. This

169 pressure is similar to the lowest pressures at which HDA ice is formed. Thus, water

170 aggregates of a few nanometres in diameter may be formed with high internal density.

171 Another possibility is that the stress in the crystalline $\alpha$-CO matrix plays an important

172 role, as is acknowledged for thin films [32]. The stress in the solid could force the water

173 molecules to be segregated into high-density pores.

174 The matrix sublimation method developed in the present study is a novel means of

175 forming amorphous ice and supplements previously known methods. In vapor

176 deposition and liquid water quenching, the surface temperature of the amorphous ice 
177 becomes higher owing to the heat of condensation [33] and solidification, respectively,

178 which can sometimes lead to the crystallisation. Conversely, in the matrix sublimation

179 method, the sample surface is cooled owing to the sublimation heat of the matrix. In the

180 high-pressure compression of ice crystals, transformation to amorphous ice is limited at

181 temperatures higher than $77 \mathrm{~K}$, and in irradiation methods, decomposition of water

182 molecules is unavoidable. However, in the matrix sublimation method, no

183 decomposition of water molecules occurs, and there is no temperature limit if we

184 choose a suitable matrix species.

185 It may be possible to easily make other amorphous and crystalline ices that usually

186 require a wide pressure range using modifications of the present matrix sublimation

187 method with, for example, various ratios of matrix to water, various types of matrix (i.e.

188 those formed at different sublimation temperatures or with different polarity materials),

189 and various matrix sublimation rates. To explore the effect of the matrix to water ratio,

190 we performed experiments using ices formed at $\mathrm{CO}: \mathrm{H}_{2} \mathrm{O}$ ratios of 10:1, 50:1 and 2:1,

191 and ASW. A comparison of the d-spacing in the MSIs prepared using these ratios is

192 presented in Supplementary Fig. 3. It is clear that the size of the d-spaces in the MSI 
193 prepared using a CO: $\mathrm{H}_{2} \mathrm{O}$ ratio of 2:1 lies between those of MS-HDA ice and ASW,

194 suggesting that the MSI prepared using the 2:1 ratio is a mixture of MS-HDA ice and

195 ASW. We also performed experiments using $\mathrm{N}_{2}$ for the matrix instead of $\mathrm{CO}$, and

196 similar results are obtained, although the d-spaces are slightly larger (Supplementary

197 Fig. 4). These results clearly indicate that the interaction between the water and matrix

198 molecules plays an essential role in determining the structure of the ice formed.

199 The most prominent feature of the matrix sublimation method is that high-pressure

200 phases are formed very easily in a vacuum, whereas highly energetic processes such as

201 ionization or high-temperature heating are required for other low-pressure syntheses,

202 such as those of diamond or cubic boron nitride. Furthermore, this method may be

203 applied to prepare other crystalline and amorphous solids. In fact, any laboratory that

204 currently performs matrix isolation spectroscopy could also perform matrix sublimation

205 preparation, as the apparatus needed for these techniques is essentially the same.

206 Consequently, rapid progress in research into the formation of new materials is now

207 possible. 


\section{Astrophysical implications}

210

211 This study also has astrophysical implications, as CO-rich ices have been observed

212 in icy grains within interstellar molecular clouds [34]. These grains consist of a silicate

213 core, an inner mantle of organic refractory material, $\mathrm{H}_{2} \mathrm{O}$-rich amorphous ice, and a

214 CO-rich outer mantle [35]. When CO sublimes due to the slight temperature increase

215 during the formation of protoplanetary disks, highly porous MS-HDA ice is formed.

216 The high porosity of icy grains could have a significant effect on the aggregation of

217 interstellar grains and the physical properties of planetesimals. Therefore, the thermal

218 conductivity of cometary nuclei might be smaller than has been thitherto thought [33].

219 Furthermore, the high porosity characteristics of cometary nuclei as observed by the

220 Rosetta mission [36] could be explained by the aggregation of highly porous

221 MS-HDA-covered icy grains. $\mathrm{CO}$ or $\mathrm{N}_{2}$-rich ices are also observed on the surface of

222 dwarf planets and icy satellites [37,38]. If CO or $\mathrm{N}_{2}$ sublimes from these ices upon

223 slight temperature increase, highly porous MS-HDA ice might be formed on the surface

224 of these bodies. 
227 References

228 [1] V.F. Petrenko, R.W. Whitworth, Physics of Ice. Oxford Univ. Press, 1999.

229 [2] C.A. Angell, Ann. Rev. Phys. Chem. 55 (2004) 559.

230 [3] A. Cavalier, D. Spehner, B.M. Humbel, eds. Handbook of Cryo-Preparation

231 Methods for Electron Microscopy. CRC Press, 2009.

232 [4] R.M.E. Mastrapa, W.M. Rundy, M.S. Gudipati, In: The Science of Solar System Ices

233 (eds. M.S. Gudipati, J.Castillo-Rogez) p371 Springer, 2013.

234 [5] E.F. Burton, W.F. Oliver, Proc. R. Soc. Lond. A153 (1935) 166.

235 [6] P. Jenniskens, D.F. Blake, Science 265 (1994) 753.

236 [7] A.H. Narten, C.G. Vekatesh, J.A. Rice, J. Chem. Phys. 64 (1976) 1106.

237 [8] E. Mayer, R. Pletzer, Nature 319 (1986) 298.

238 [9] K.P. Stevenson, G.A. Kimmel, Z. Dohnalek, R.S. Smith, B.D. Kay, Science 283

239 (1999) 1505.

240 [10] P. Brüggeller, E. Mayer, Nature 288 (1980) 569. 
241 [11] J. Dubochet, A.W. McDowell, J. Microsc. 124 (1981) RP3.

242 [12] O. Mishima, Y. Suzuki, J. Chem. Phys. 115 (2001) 4199.

243 [13] O. Mishima, L.D. Calvert, E. Whalley, Nature 310 (1984) 393.

244 [14] T. Loerting, C.G. Salzmann, I. Kohl, E. Mayer, A. Hallbrucker, Phys. Chem. Chem.

245 Phys. 3 (2001) 5355.

246 [15] J. Lepault, R. Freeman, J. Dubochet, J. Microsc. 132 (1983) RP3.

247 [16] A. Kouchi, T. Kuroda, Nature 344 (1990) 134.

248 [17] O. Mishima, H.E. Stanley, Nature 396 (1998) 329.

249 [18] K. Winkel, M.S. Elsaesser, E. Mayer, T. Loerting, J. Chem. Phys. 128 (2008)

$250 \quad 044510$

251 [19] C.A. Angel, Science 319 (2008) 582.

252 [20] Y. Kondo, K. Ohi, Y. Ishibashi, H. Hirano, Y. Harada, K. Takayanagi,

253 Ultramicroscopy 35 (1991) 111.

254 [21] N. Watanabe, T. Shiraki, A. Kouchi, Astrophys. J. Lett. 588 (2003) L121.

255 [22] W. Hagen, A.G.G.M. Tielens, J.M. Greenberg, Chem. Phys. 56 (1981) 367.

256 [23] A. Givan, A. Loewenschuss, C.J. Nielsen, J. Chem. Soc. Faraday Trans. 92 (1996) 
258 [24] A. Givan, A. Loewenschuss, C.J. Nielsen, Vib. Spectrosc. 16 (1998) 85.

259 [25] C.G. Salzmann, T. Loerting, S. Klotz, P.W. Mirwald, A. Hallbrucker, E. Mayer, 260 Phys. Chem. Chem. Phys. 8 (2006) 386.

261 [26] T. Loerting, C.G. Salzmann, K. Winkel, E. Mayer, Phys. Chem. Chem. Phys. 8 262 (2006) 2810.

263 [27] O. Mishima, J. Chem. Phys. 100 (1994) 5910.

264 [28] P.H. Handle, M. Seidl, T. Loeting, Phys. Rev. Lett. 108 (2012) 225901.

265 [29] M. Seidl, K. Amann-Winkel, P.H. Handle, G. Zifferer, T. Loerting, Phys. Rev. B 88 266 (2013) 174105.

267 [30] W. Hagen, A.G.G.M. Tielens, J.M. Greenberg, Chem. Phys. 56 (1981) 367.

268 [31] V. Buch, J.P. Devlin, J. Chem. Phys. 94 (1991) 4091.

269 [32] F. Spaepen, Acta Mater. 48 (2000) 31.

270 [33] A. Kouchi, J.M. Greenberg, T. Yamamoto, T. Mukai, Astrophys. J. Lett. 388 (1992)

$271 \quad$ L73.

272 [34] W.F. Thi, K.M. Pontoppidan, E.F. van Dishoeck, E. Dartois, L. d’Hendecourt, 
273 Astron. Astrophys. 394 (2002) L27.

274 [35] M. Spaans, P. Ehrenfreund, In: Laboratory Astrophysics and Space Research, eds. P.

275 Ehrenfreund, K. Krafft, H. Kochan, V. Pirronello, Kluwer Academic Publisher, p. 1 276 (1999).

277 [36] W. Kofman, et al. Science 349 (2015) aab0639-1.

278 [37] T.C. Owen, T.L. Roush, D.P. Cruikshank, J.L. Elliot, L.A. Young, C. de Bergh, B. 279 Schmitt, T.R. Geballe, R.H. Brown, M.J. Bartholomew, Science 261 (1993) 745.

280 [38] W.M. Grundy, L.A. Young, J.A. Stansberry, M.W. Buie, C.B. Olkin, E.F. Young, 281 Icarus 205 (2010) 594.

\section{Ackowledgements}

284 We thank O. Mishima for critical reading of the manuscript, and S. Nakatsubo, K.

285 Fujita, K. Sinbori and M. Ikeda for their help in the development of UHV-TEM. This 286 study was supported by the Grant for the Joint Research Program of the Institute of Low 287 Temperature Science, Hokkaido University, I-link-1027 project from CSIC, JSPS 288 KAKENHI (Grant Number 25247086), and MEXT KAKENHI (Grant Number 
25108002).

292 Figure captions

293

294 Figure 1. Temperature-density diagram schematic of the formation of amorphous ices.

295 The starting materials are shown in rounded rectangles, formation routes as arrows with

296 names of methods, and products in rectangles. $\mathrm{T}_{\mathrm{g}}$ is the glass transition temperature.

297 HD-ASW, high-density amorphous solid water; LD-ASW, low-density amorphous solid

298 water; HGW, hyperquenched glassy water; HDA, high-density amorphous ice; HD,

299 high density; LD, low density; MS-HDA, matrix-sublimated high-density amorphous 300 ice; Ih, hexagonal ice.

301

302 Figure 2. Matrix sublimation method. Deposition of $\mathrm{CO}: \mathrm{H}_{2} \mathrm{O}$ on an 8-10 K substrate in

303 an ultra-high vacuum chamber. After deposition, the substrate is warmed at a constant

304 rate. Sublimation of matrix CO at $25-40 \mathrm{~K}$. Formation of MSI at T $>40 \mathrm{~K}$. 
306 Figure 3. Low-magnification TEM image and corresponding electron diffraction 307 pattern of initial $\mathrm{CO}: \mathrm{H}_{2} \mathrm{O}=10: 1$ ice at $8 \mathrm{~K}$ (a), and MS-HDA at $80 \mathrm{~K}$ (b). Electron 308 diffraction pattern was obtained from the central circle region of $0.6 \mu \mathrm{m}$ diameter.

310 Figure 4. Textural variety in highly porous MS-HDA ice. Generally, the black part is 311 thicker and white part thinner. (a, b) Initial CO: $\mathrm{H}_{2} \mathrm{O}=50: 1$. (c, d) Initial CO: $\mathrm{H}_{2} \mathrm{O}=10: 1$. 312 (Observation temperatures: a, 43 K; b, 36 K; c, 87 K; and d, 34 K.)

314 Figure 5. Low-magnification TEM image and corresponding electron diffraction 315 pattern of vapor-deposited ASW at $10 \mathrm{~K}$.

317 Figure 6. Temperature dependence of d-spacing in the main halo of the electron 318 diffraction patterns of amorphous ices. ASW (orange circles) and MS-HDA ice (blue squares: $\mathrm{CO}: \mathrm{H}_{2} \mathrm{O}=10: 1$, green diamonds: $\mathrm{CO}: \mathrm{H}_{2} \mathrm{O}=50: 1$ ). 
321 Figure 7. Transformation from MS-HDA to ice Ic. Low-magnification TEM image and corresponding electron diffraction pattern of MS-HDA ice and ice Ic at $136 \mathrm{~K}$.

324 Figure 8. Reflection-absorption IR spectra at different temperatures. (a, b) Initial composition of $\mathrm{CO}: \mathrm{H}_{2} \mathrm{O}=50: 1$. (c, d) Initial composition of $\mathrm{CO}: \mathrm{H}_{2} \mathrm{O}=10: 1$. (e, f) Pure $326 \mathrm{H}_{2} \mathrm{O}$. b, d, and f show the change in the $\mathrm{OH}$ stretching bands between 10 and $50 \mathrm{~K}$.

Table 1. Assignment of water clusters in CO matrix.

333 Figure 1. Spectral change of water clusters in $\mathrm{CO}: \mathrm{H}_{2} \mathrm{O}$ ices from 10 to $30 \mathrm{~K}$. (a) Initial

$334 \mathrm{CO}: \mathrm{H}_{2} \mathrm{O}=50: 1$ (b) Initial $\mathrm{CO}: \mathrm{H}_{2} \mathrm{O}=10: 1$. Wavenumbers marked by dotted lines are 335 the same as those in Supplementary Table 1. Red and blue lines indicate monomers and polymers, respectively. 
338 Figure 2. Reflection-absorption IR spectra at 50 K. (a) Initial composition of CO: $\mathrm{H}_{2} \mathrm{O}$

$339=50: 1$. (b) Initial composition of CO: $\mathrm{H}_{2} \mathrm{O}=10: 1$. (c) Vapor-deposited $\mathrm{H}_{2} \mathrm{O}$ (ASW).

340 Peaks at 3698 and $3696 \mathrm{~cm}^{-1}$ indicate dangling-OH bonds.

342 Figure 3. Temperature dependence of the d-spacing in the main halo of the electron

343 diffraction patterns of amorphous ices made from CO: $\mathrm{H}_{2} \mathrm{O}=2: 1$ (yellow triangles).

344 Grey symbols (circles, diamonds and squares) are the same data as presented in Fig. 5.

346 Figure 4. Temperature dependence of the d-spacing in the main halo of the electron

347 diffraction patterns of MS-HDA ice (blue stars: $\mathrm{N}_{2}: \mathrm{H}_{2} \mathrm{O}=10: 1$; green hexagons:

$348 \mathrm{~N}_{2}: \mathrm{H}_{2} \mathrm{O}=50: 1$ ). Grey symbols (circles, diamonds and squares) are the same data as in 349 Fig. 5. 


\section{$\mathrm{H}_{2} \mathrm{O} \quad \mathrm{CO}$}

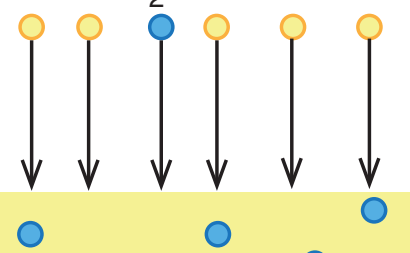

$\mathrm{CO}$

matrix 0

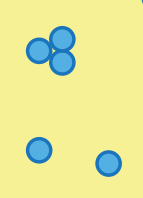

- $\infty$

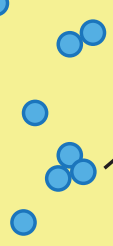

substrate

Fig. 2

$8-10 \mathrm{~K}$

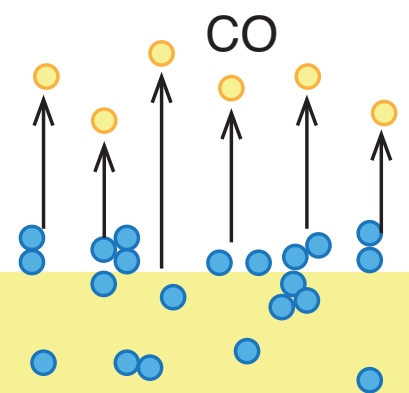

MSI
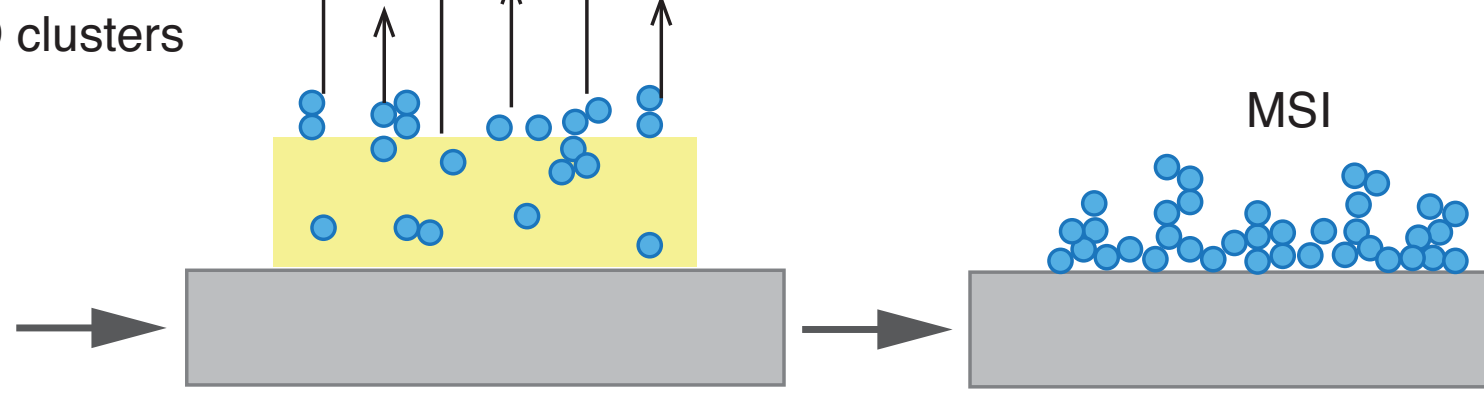

O

$25-40 \mathrm{~K}$

$>40 \mathrm{~K}$ 


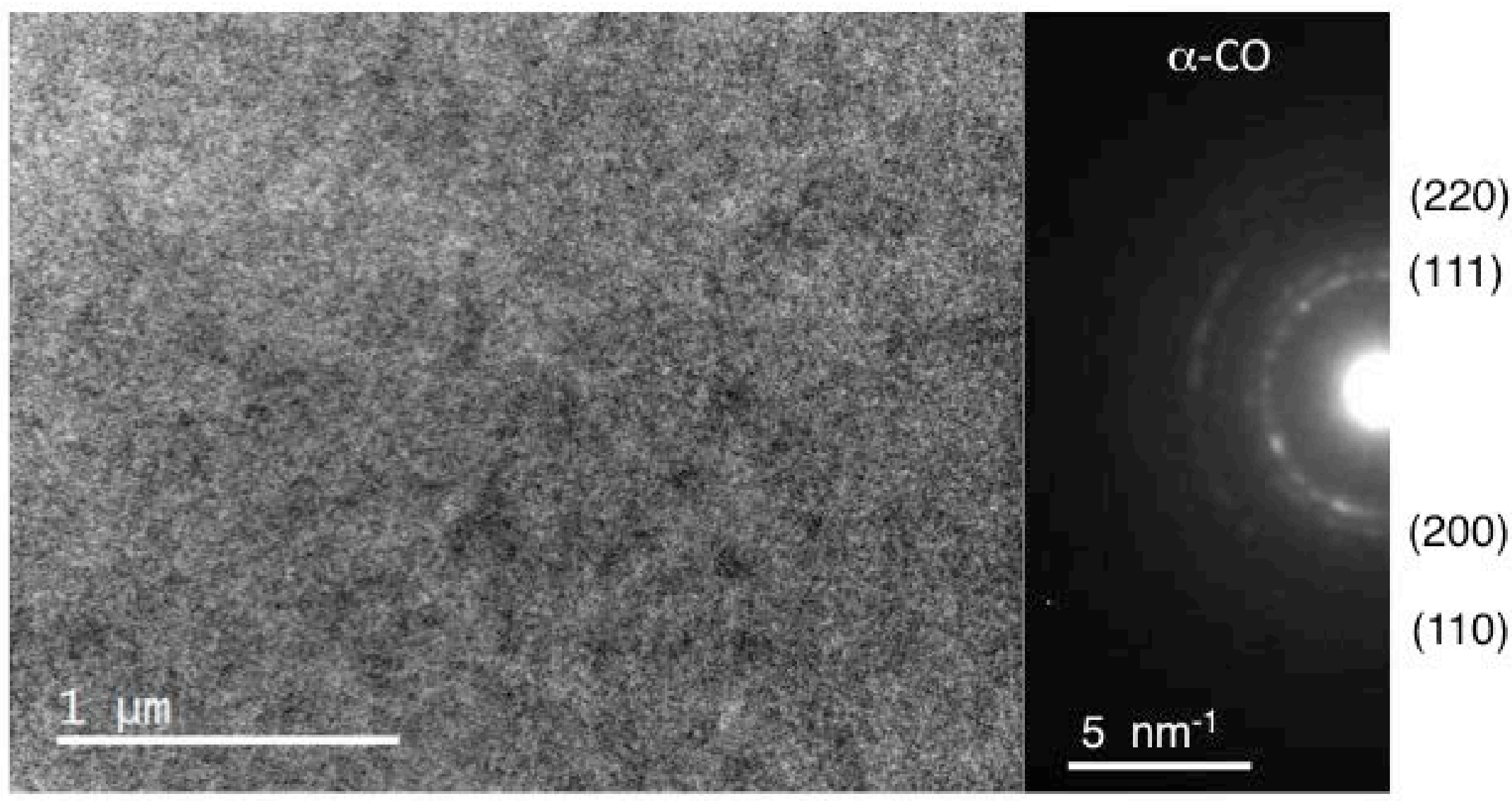

Fig. 3a 


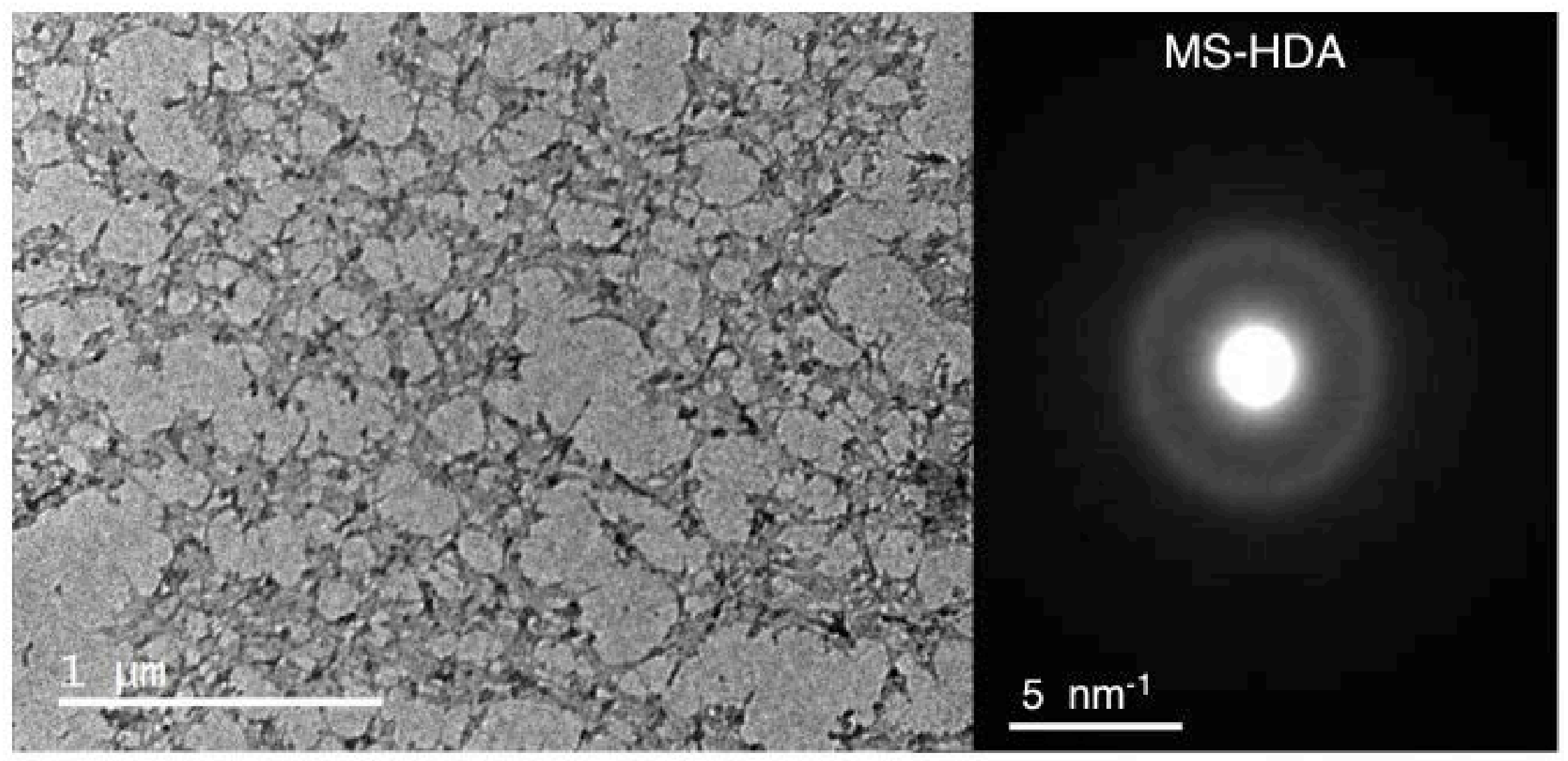

Fig. 3b 

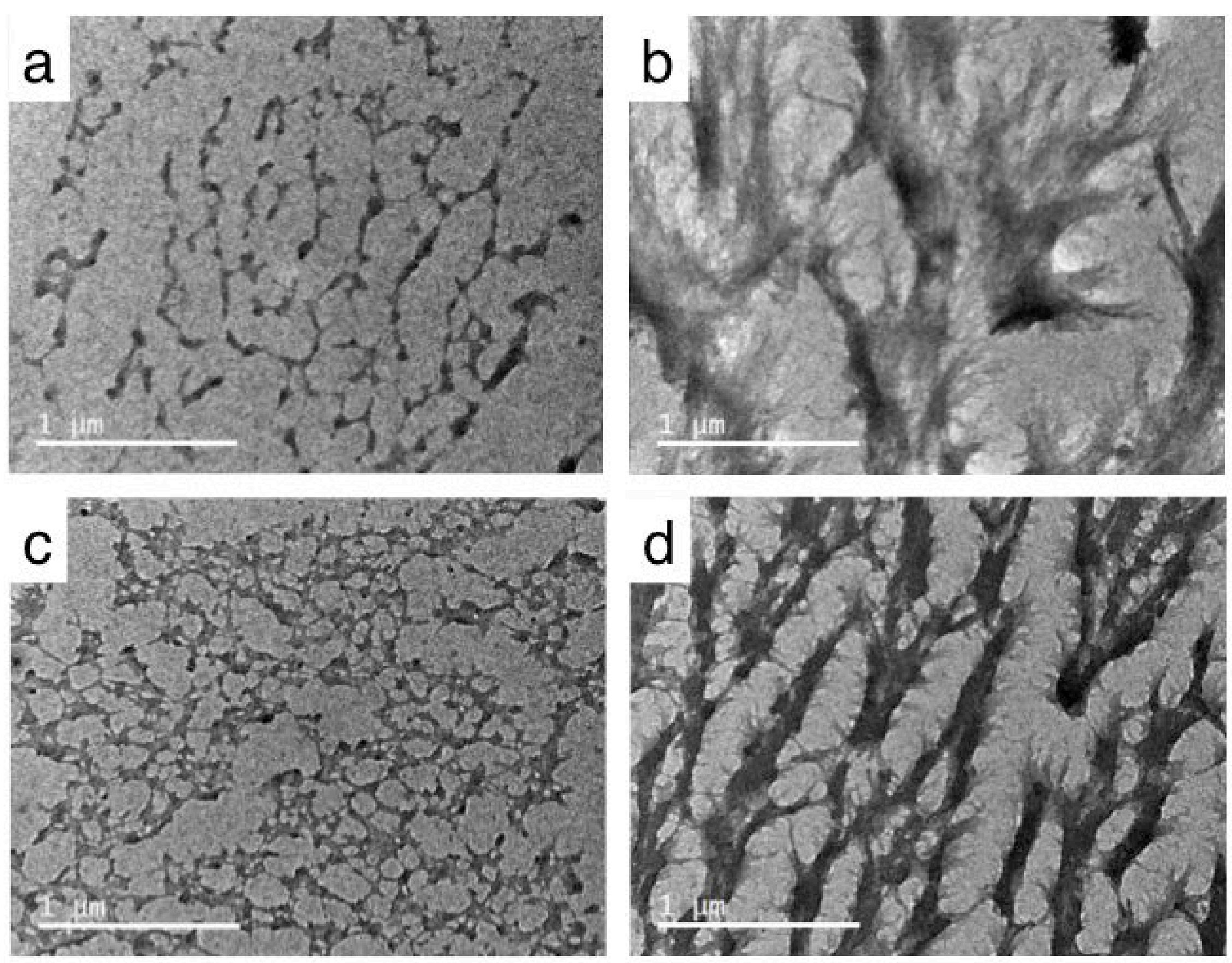


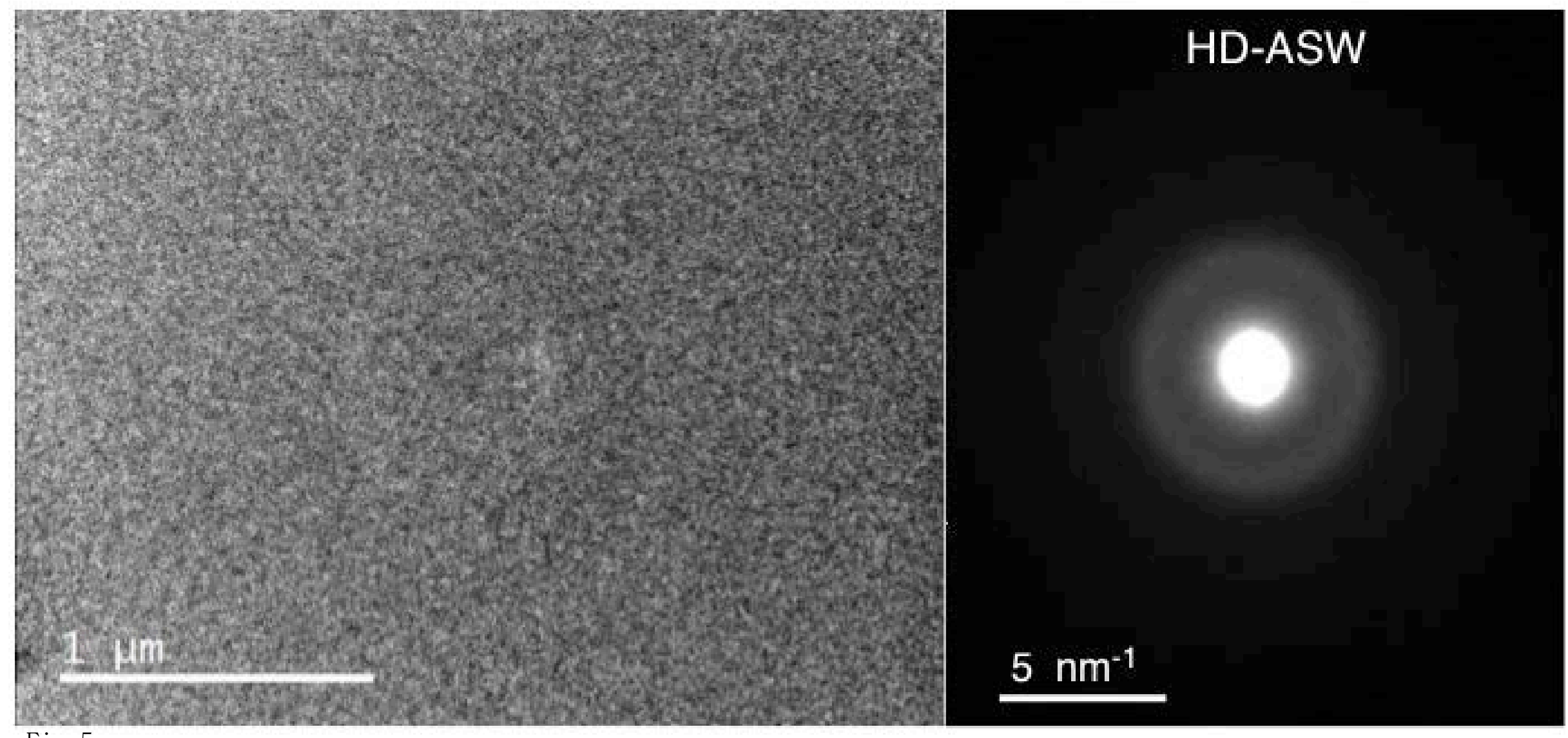

Fig. 5 


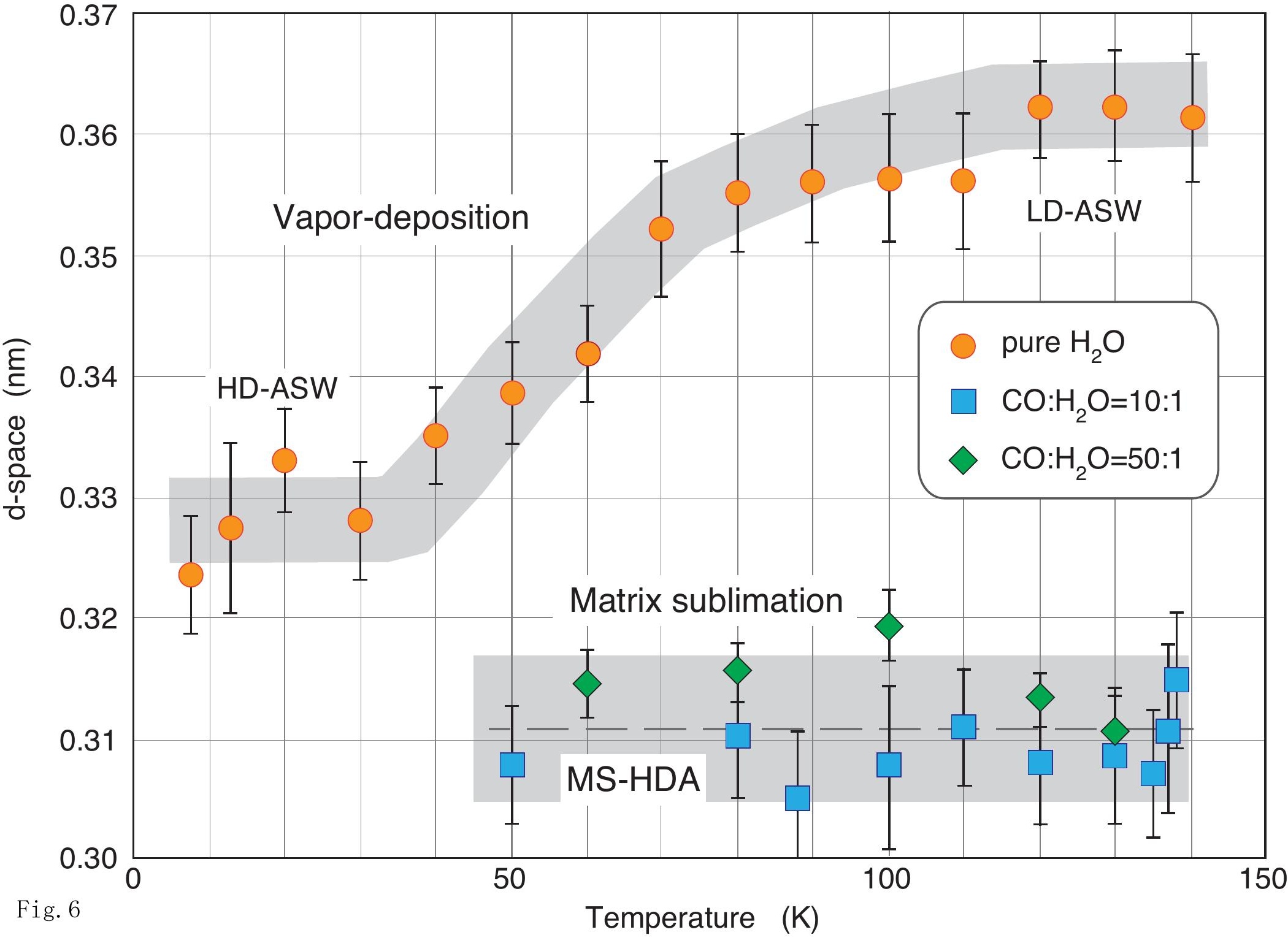




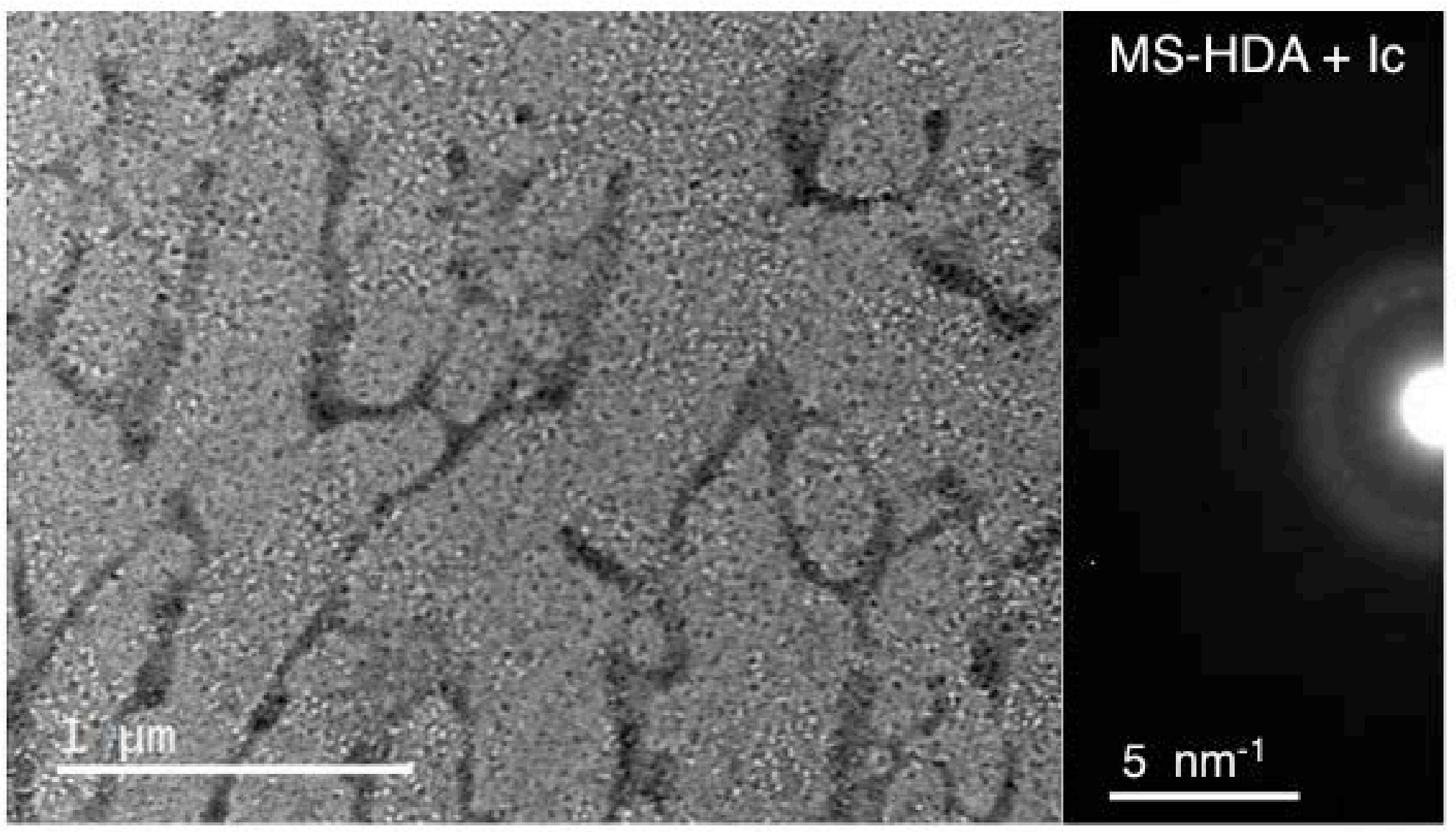

(111)

Fig. 7 

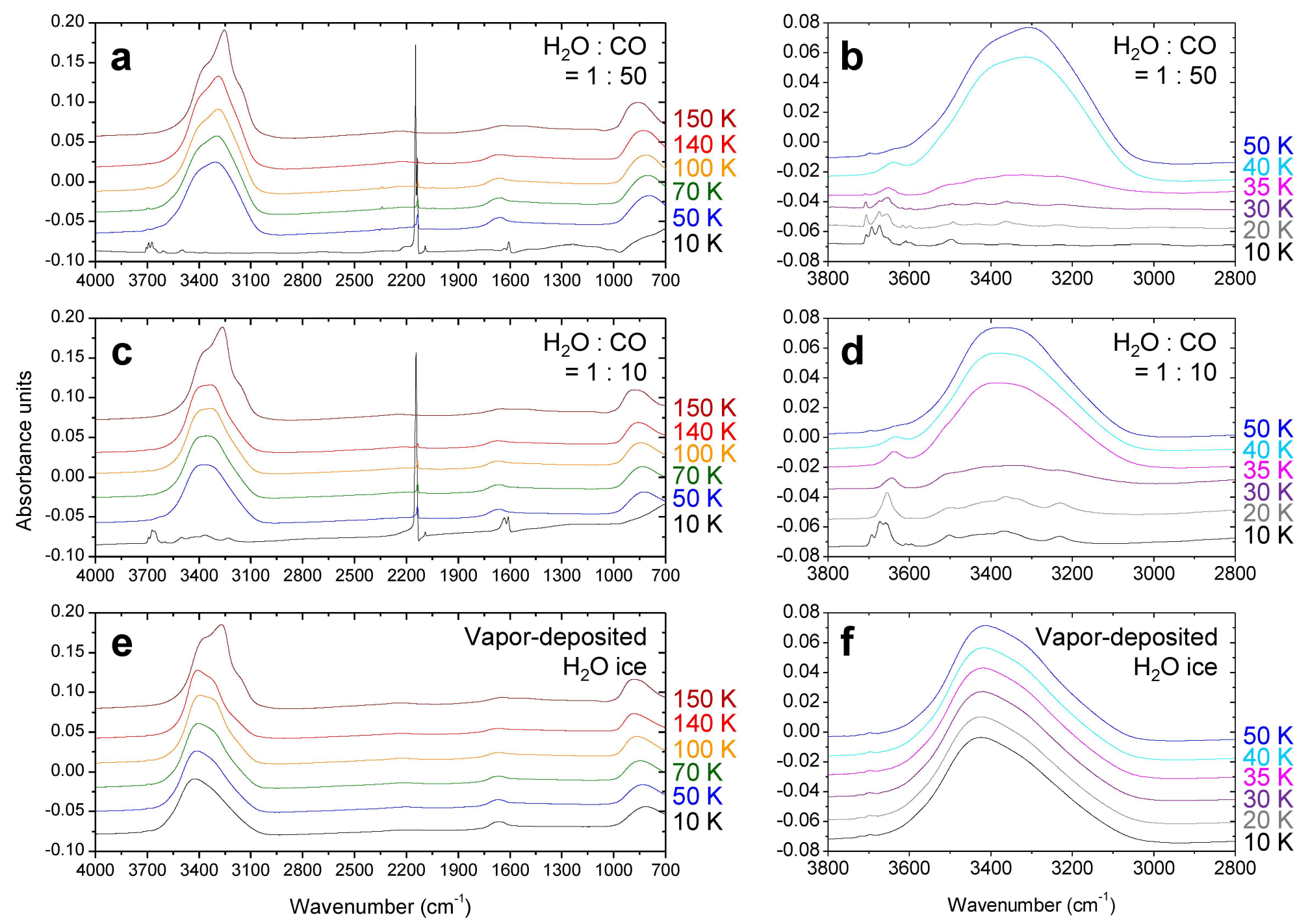

Fig. 8 
Supplementary-Table 1. Assignment of water clusters in CO matrix based on previous works.

Symbols $v_{1}$, and $v_{3}$, refer to the symmetric, and the asymmetric $\mathrm{OH}$ stretching modes, respectively. $(\mathrm{CO})_{m}\left(\mathrm{H}_{2} \mathrm{O}\right)_{n}$ represents complexes of $\mathrm{CO}$ and $\mathrm{H}_{2} \mathrm{O}$.

\begin{tabular}{|c|c|c|c|}
\hline Wavenumber $\left(\mathrm{cm}^{-1}\right)$ & Assignment & Mode & References $^{1}$ \\
\hline \multirow[t]{2}{*}{3707} & Monomer & $v_{3}$ & {$[22]$} \\
\hline & & $v_{3}$ for monomeric $\mathrm{H}_{2} \mathrm{O}$ trapped in & \\
\hline \multirow[t]{3}{*}{3692} & Monomer & a different matrix site. & {$[22]$} \\
\hline & $(\mathrm{CO})_{m}\left(\mathrm{H}_{2} \mathrm{O}\right)_{n}$ & $v_{3}$ & {$[23]$} \\
\hline & $\mathrm{CO}-\mathrm{H}_{2} \mathrm{O}$ ice interface, or Dimer & OH stretching & {$[24]$} \\
\hline \multirow[t]{3}{*}{3674} & Dimer & $v_{3}$ & {$[22]$} \\
\hline & $(\mathrm{CO})_{m}\left(\mathrm{H}_{2} \mathrm{O}\right)_{n}$ & $v_{3}$ & {$[23]$} \\
\hline & $\mathrm{CO}-\mathrm{H}_{2} \mathrm{O}$ ice interface, or Dimer & OH stretching & {$[24]$} \\
\hline 3658 & Tetramer, Polymer & OH stretching & {$[22]$} \\
\hline 3640 & Polymer & OH stretching & {$[22]$} \\
\hline 3617 & Monomer & $v_{1}$ & {$[22]$} \\
\hline 3610 & Dimer & OH stretching & {$[24]$} \\
\hline 3598 & Dimer, Polymer & $v_{1}$ & {$[22]$} \\
\hline 3495 & Dimer & $v_{3}$ & {$[22]$} \\
\hline 3438 & Trimer & OH stretching & {$[22]$} \\
\hline
\end{tabular}


${ }^{1}$ : [22] Hagen et al. (1981), [23] Givan et al. (1996), [24] Givan et al. (1998) 

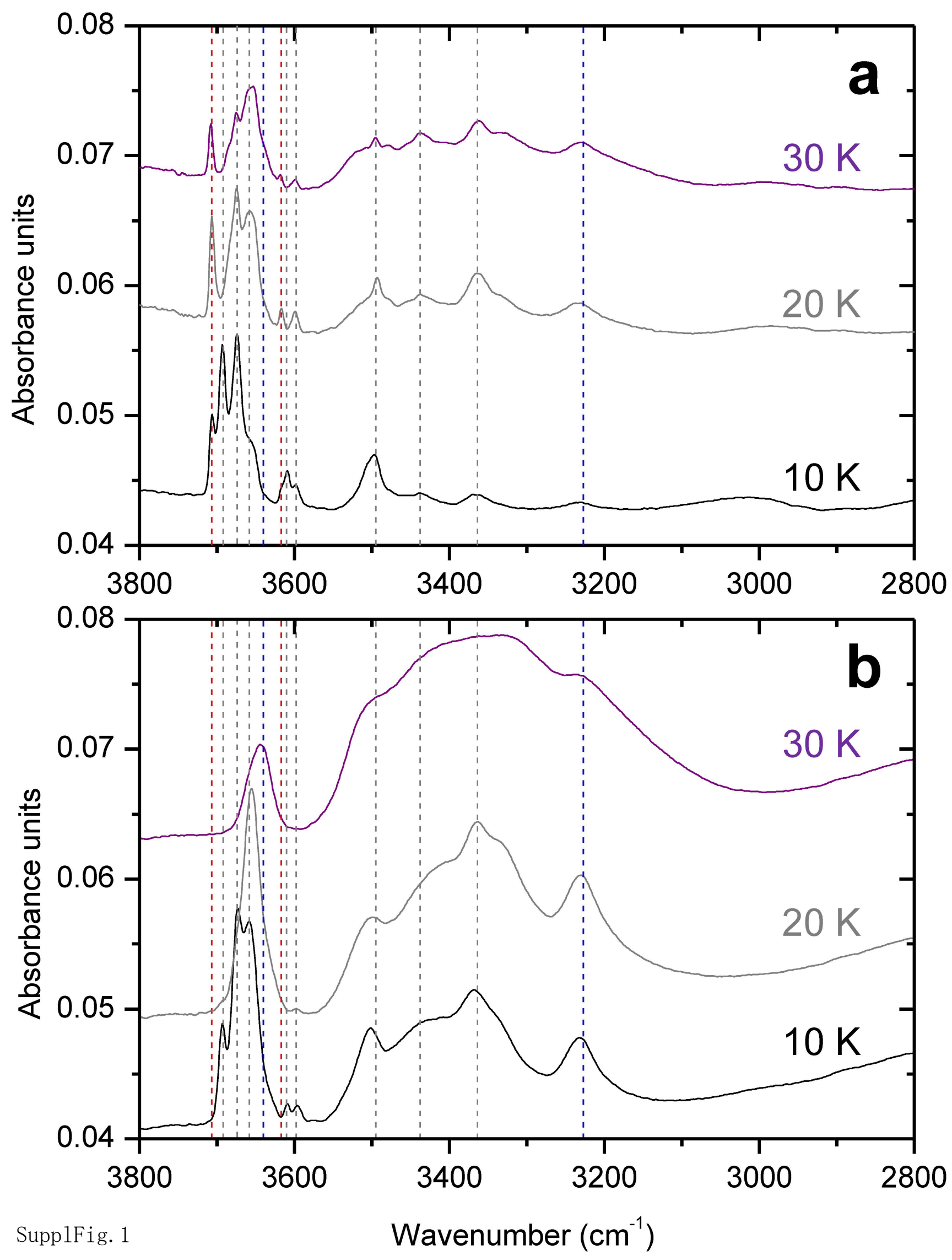

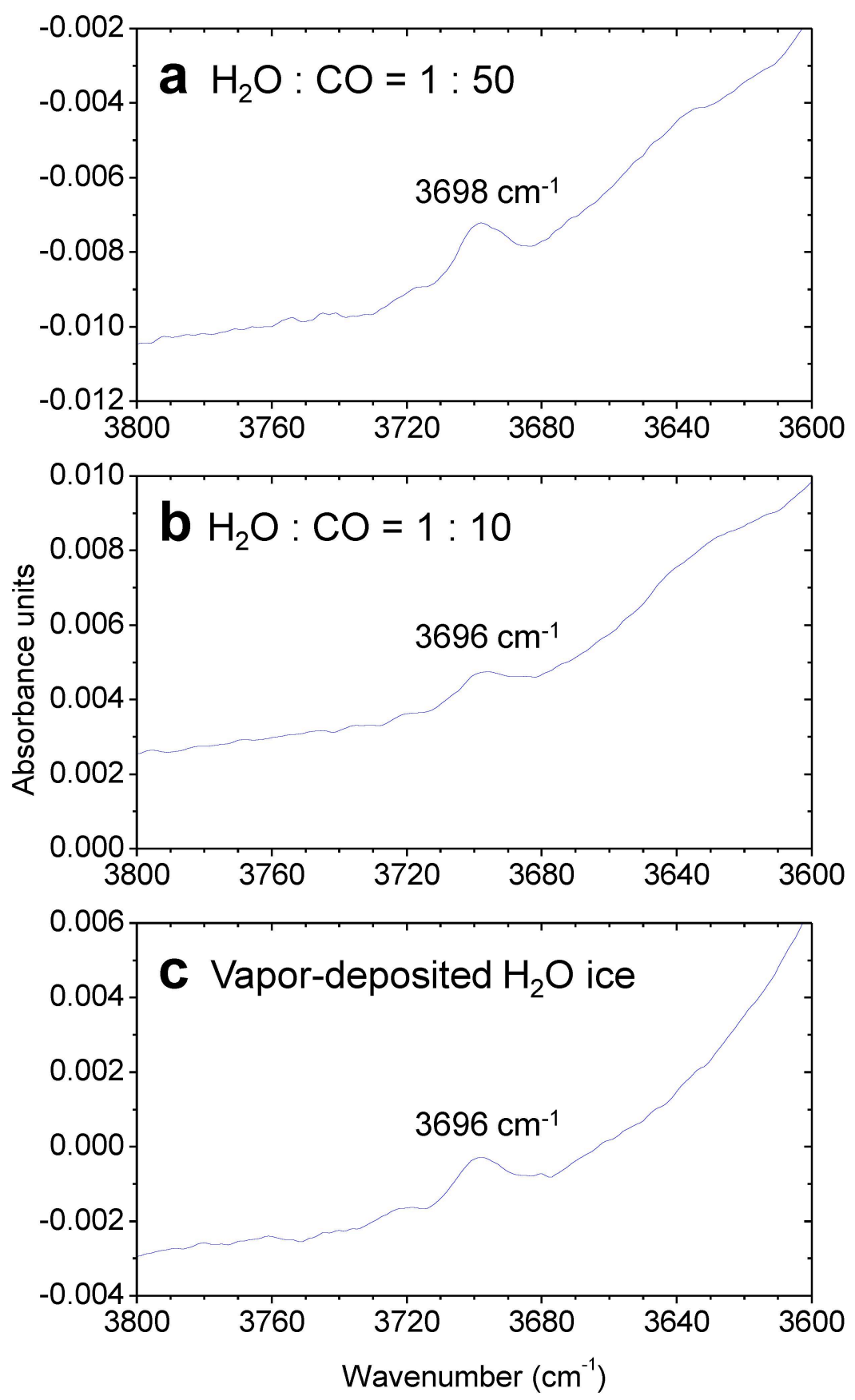


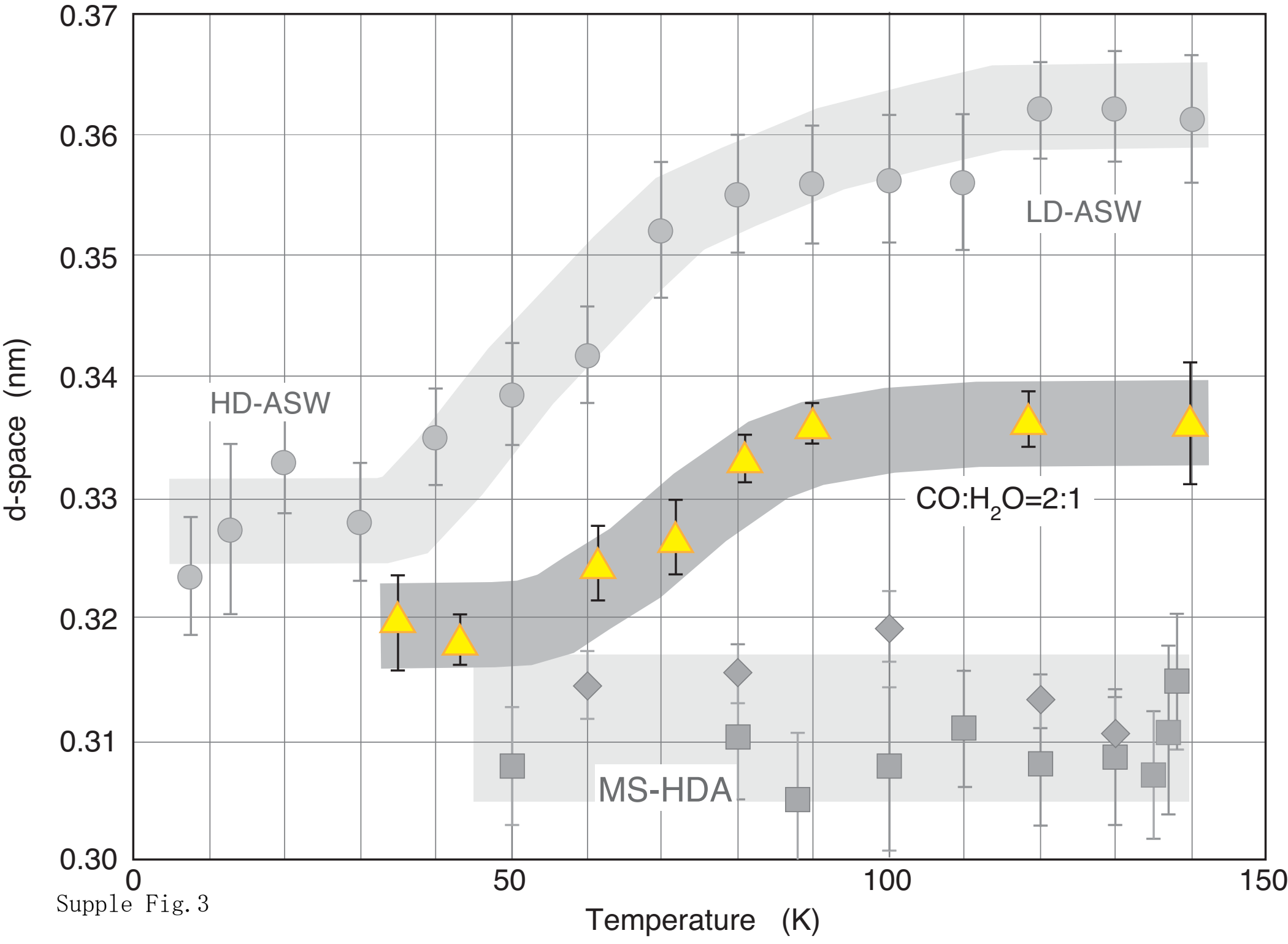




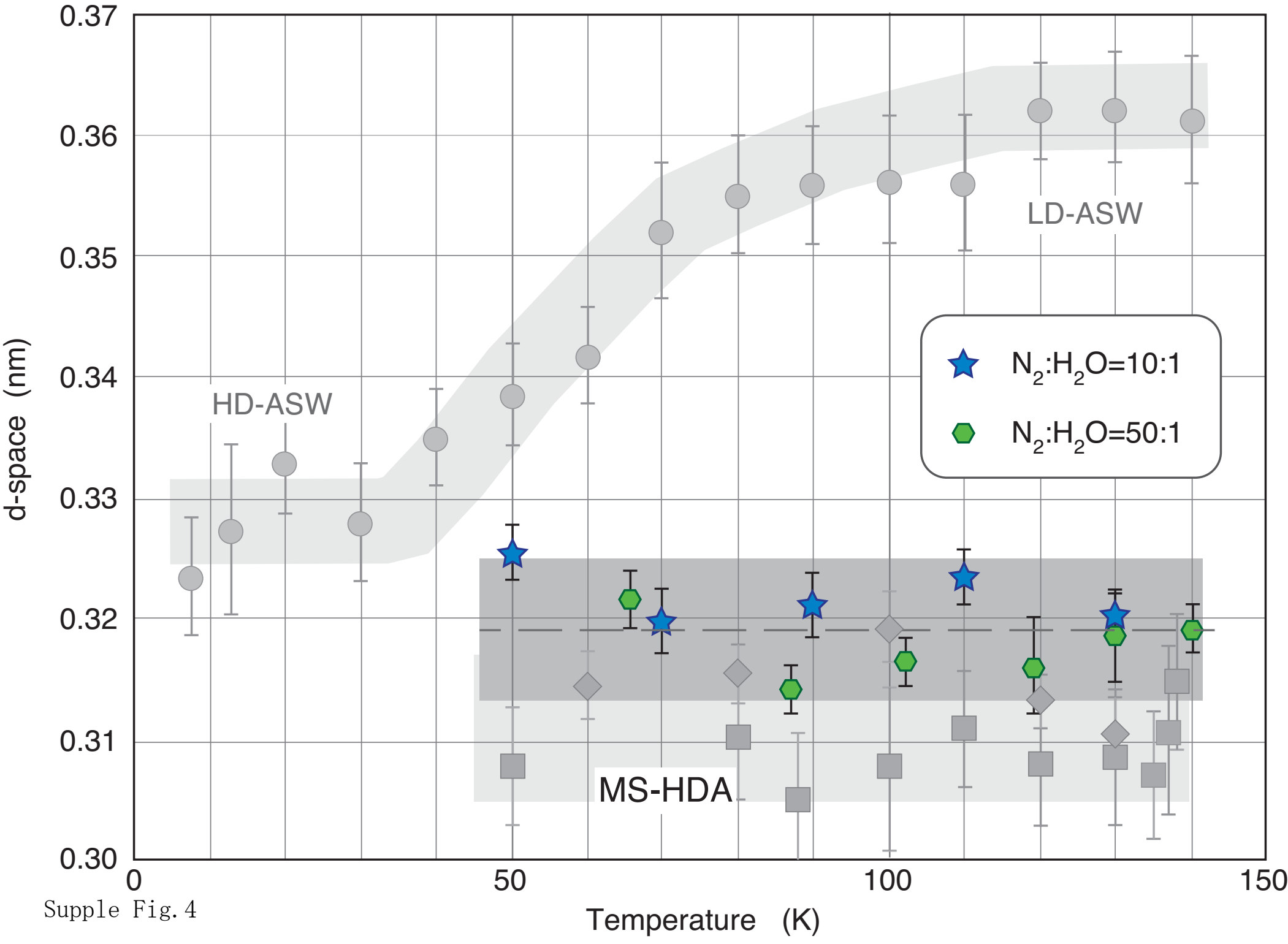

